https://doi.org/10.15407/ujpe66.12.1024

B.E. GRINYUK, K.A. BUGAEV

Bogolyubov Institute for Theoretical Physics, Nat. Acad. of Sci. of Ukraine

(14-B, Metrolohychna Str., Kyiv 03143, Ukraine; e-mail: bgrinyuk@bitp.kiev.ua)

\title{
ABOUT CONDITIONS OF SPATIAL COLLAPSE IN AN INFINITE SYSTEM OF BOSE PARTICLES
}

\begin{abstract}
Using the variational principle, we show that the condition of spatial collapse in a Bose gas is not determined by the value of the scattering length of the interaction potential between particles contrary to the result following from the Gross-Pitaevskii equation, where the collapse should take place at a negative scattering length.
\end{abstract}

Ke ywords: Bose-system, spatial collapse.

\section{Introduction}

The spatial collapse of an infinite system of interacting Bose particles was commonly analyzed within the approach based on the Gross-Pitaevskii [1-4] equation (see also the latest studies [5]). An obvious result of this analysis lies in the fact that, under the condition $a<0$ [6] (where $a$ is the two-particle scattering length determining the sign of non-linear term of the equation), one has a collapse in the system.

In the present paper, starting from the initial Hamiltonian of a system of $N$ Bose particles and using the variational principle, we show that the spatial collapse of the Bose system at $N \rightarrow \infty$, generally speaking, is not determined by the two-particle scattering length value.

\section{Statement of the Problem}

We consider a system of $N$ identical interacting Bose particles of mass $m$ with the Hamiltonian

$\hat{H}=\sum_{k=1}^{N} \frac{\hat{\mathbf{p}}_{k}^{2}}{2 m}+\sum_{n>k=1}^{N} V\left(\left|\mathbf{r}_{\mathbf{n}}-\mathbf{r}_{\mathbf{k}}\right|\right)$,

where the pairwise potential depends on the distance between particles. In the present paper, we consider a rather wide class of potentials obeying the inequality

$V(r)<V_{0}(r) \equiv$

$\equiv V_{01} \exp \left(-\left(r / r_{01}\right)^{2}\right)-V_{02} \exp \left(-\left(r / r_{02}\right)^{2}\right)$

(c) B.E. GRINYUK, K.A. BUGAEV, 2021

\section{4}

with $r_{01}<r_{02}$ and $V_{01} \geq 0, V_{02} \geq 0$. If, for the "reference" potential $V_{0}(r)$ with a definite set of parameters, one obtains the collapse in the Bose system (1), then, for any potential $V(r)<V_{0}(r)$, the collapse will obviously take place even more. The "reference" potential is chosen in the form (2) for the convenience in order to calculate some integrals in an explicit form (see below).

Now, we are going to carry on a rather simple variational estimation of the ground state energy of the system of Bose particles with the Hamiltonian (1) and to find a sufficient condition for the spatial collapse of the system to exist. It should be stressed that the condition to be obtained will be not a necessary criterion, but only a sufficient one. In principle, one can carry out a more accurate variational estimation and obtain a more refined sufficient condition.

\section{Variational Estimation with One-Particle Trial Functions}

As is well-known (see, e.g., [7]), the ground-state energy $E_{0}$ of any quantum system does not exceed the average

$E_{0} \leq \frac{\langle\Psi|\hat{H}| \Psi\rangle}{\langle\Psi \mid \Psi\rangle}$

where $\Psi$ is an arbitrary ("trial") function. The sign of equality is valid, if $\Psi$ is the exact solution of the Schrödinger equation for the ground state of the system. Further, we will consider the Hamiltonian $\hat{H}_{0}$ with a "reference" potential $V_{0}$ of the special form (2).

ISSN 2071-0194. Ukr. J. Phys. 2021. Vol. 66, No. 12 
Consider the trial function $\Psi$ in the simplest form of a product of one-particle functions:

$$
\begin{aligned}
& \Psi\left(\mathbf{r}_{1}, \mathbf{r}_{2}, \ldots, \mathbf{r}_{N}\right)=\prod_{k=1}^{N} \exp \left(-\left(\mathbf{r}_{k} / R\right)^{2}\right) \equiv \\
& \equiv \exp \left(-\frac{1}{R^{2}} \sum_{k=1}^{N} \mathbf{r}_{k}^{2}\right),
\end{aligned}
$$

where $R$ characterizes the size of the system. The choice of a trial function in the form (4), as well as the choice of a "reference" potential, enables one to calculate integrals explicitly. In particular, the normalization integral results in

$\langle\Psi \mid \Psi\rangle=\left(\frac{\pi}{2}\right)^{\frac{3 N}{2}} R^{3 N}$.

The average of the Hamiltonian consists of two terms. The first one is the matrix element of the kinetic energy

$\left\langle\Psi\left|\sum_{k=1}^{N} \frac{\hat{\mathbf{p}}_{k}^{2}}{2 m}\right| \Psi\right\rangle \equiv-\frac{\hbar^{2}}{2 m} \sum_{k=1}^{N}\left\langle\Psi\left|\triangle_{k}\right| \Psi\right\rangle=$

$=N \frac{3 \hbar^{2}}{2 m R^{2}}\left(\frac{\pi}{2}\right)^{\frac{3 N}{2}} R^{3 N}$,

and the second one is the potential energy matrix element:

$\left\langle\Psi\left|\sum_{n>k=1}^{N} V_{0}\left(\left|\mathbf{r}_{n}-\mathbf{r}_{k}\right|\right)\right| \Psi\right\rangle=$

$=\frac{N(N-1)}{2} \frac{1}{R^{3}}\left(V_{01} \rho_{01}^{3}-V_{02} \rho_{02}^{3}\right)\left(\frac{\pi}{2}\right)^{\frac{3 N}{2}} R^{3 N}$,

where $\rho_{0 j} \equiv r_{0 j}\left(1+r_{0 j}^{2} / R^{2}\right)^{-1 / 2}, j=1,2$. If parameter $R$ is essentially greater than the radius of forces, $R \gg r_{0 j}$, one has $\rho_{0 j} \rightarrow r_{0 j}$.

Using integrals (5)-(7) in the explicit form, we obtain the following estimation for the ground-state energy of the Hamiltonian $\hat{H}_{0}$ :

$E_{0} \leq N \frac{3 \hbar^{2}}{2 m R^{2}}+\frac{N(N-1)}{2} \frac{1}{R^{3}}\left(V_{01} \rho_{01}^{3}-V_{02} \rho_{02}^{3}\right)$,

or, for a finite $R$, but $R \gg r_{0 j}$, and divided by the number of particles,

$\frac{E_{0}}{N} \leq \frac{3 \hbar^{2}}{2 m R^{2}}+\frac{N-1}{2} \frac{1}{R^{3}}\left(V_{01} r_{01}^{3}-V_{02} r_{02}^{3}\right)$.

It obviously follows from estimation (9) that, under the condition

$V_{01} r_{01}^{3}-V_{02} r_{02}^{3}<0$, the energy per one particle (9) tends to $-\infty$, as $N \rightarrow \infty$. In addition, the less the parameter $R$, the lower is the energy due to the $\sim R^{-3}$ dependence of the potential energy as compared to the $\sim R^{-2}$ dependence of the kinetic energy. Thus, one has a spatial collapse of the system of Bose particles to a small area of the order of the radius of forces (or a little bit greater) with an infinite density and an infinite negative energy even calculated per one particle.

\section{Discussion}

Since the integral over the "reference" potential $V_{0}(r)$ equals

$\int V_{0}(r) d \mathbf{r}=\pi^{3 / 2}\left(V_{01} r_{01}^{3}-V_{02} r_{02}^{3}\right)$,

one can use the condition

$\int V_{0}(r) d \mathbf{r}<0$

instead of (10). It is well known that the two-particle scattering length $a$ is expressed through the scattering amplitude at the zero transferred momentum as $a=-\left.(k \cot \delta(k))^{-1}\right|_{k \rightarrow 0}$, and, in the Born approximation, $a_{\text {Born }}$ is proportional to the integral over the potential [7]

$a_{\text {Born }}=\frac{\mu}{2 \pi \hbar^{2}} \int V_{0}(r) d \mathbf{r}$,

where $\mu$ is the reduced mass of two particles $(\mu=m / 2$ for identical particles). Thus, condition (12) can be simply rewritten as $a_{\text {Born }}<0$, where the scattering length in the Born approximation is calculated for the "reference" potential $V_{0}(r)$. Moreover, it can be shown for any short-range potential $V(r)$ that the condition like (12) or $a_{\text {Born }}<0$ is valid, if the parameter $R$ in function (4) is essentially greater than the radius of forces (but remains to be finite in order to we can speak about spatial collapse).

It is important to emphasize, that more accurate variational estimations will never lead us to the condition of collapse like $a<0$, which follows from the Gross-Pitaevskii equation (where $a$ is the exact scattering length). Really, let us start with some "reference" potential $V_{0}(r)$ giving an infinitesimal, but already negative integral (12). As a result, we already have the collapse of the system. Let us add an attraction term of the intensity $-g(g>0)$, and with 


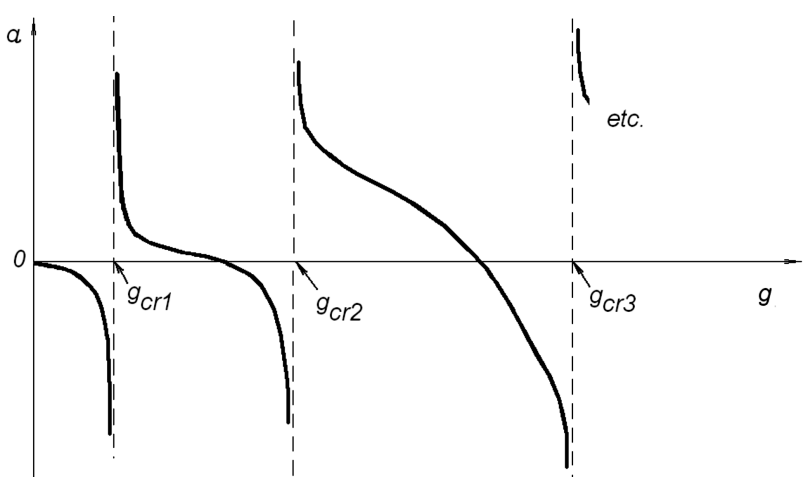

A schematic typical dependence of the scattering length $a$ on the intensity of attraction $g$

the radius $r_{02}$, to this potential. Thus, we obtain a potential $V(r)$ in the form:

$V(r) \equiv V_{0}(r)-g \exp \left(-\left(r / r_{02}\right)^{2}\right)$.

Since we have $V(r)<V_{0}(r)$, the collapse in the system of Bose particles, all the more, will take place at any $g>0$. But a dependence of the scattering length $a$ on $g$ is known to be like that shown in Figure. This dependence can be verified using the LippmannSchwinger integral equation [8] for the $t$-matrix. To find the scattering length, the variable phase approach to the potential scattering can also be used $[9,10]$, where a first-order differential equation is to be solved. In Figure, we depict a typical dependence of the scattering length on the intensity $g$ for the potential (14). At $g=0$, one has the almost zero, but negative scattering length for this potential. Near the critical values of the attraction intensity $g$ (where new two-particle bound states appear), the scattering length is known to have non-removable discontinuity, and it changes its value from minus to plus infinity. One can easily see that the scattering length may have any value and sign, but the collapse of the system is still present and even becomes stronger and stronger with an increase of $g$. Note that, at the same time, the integral over the potential and, thus, the scattering length in the Born approximation $a_{\text {Born }}$ remains to be negative, as the constant $g$ grows. Thus, the condition $a_{\text {Born }}<0$ may serve to be a sufficient condition for the collapse to take place. But we stress that it is not the necessary condition: more accurate variational estimations may show the existence of the Bose system collapse even in cases where $a_{\text {Born }}>0$.

\section{Conclusions}

To summarize, we note that the scattering length $a$ of the two-particle interacting potential cannot serve to be the value determining the presence or absence of a collapse in an infinite system of interacting Bose particles, in the contrary to the conclusions following from the Gross-Pitaevskii equation. This statement is substantiated with the use of the variational principle with the one-particle approximation for trial functions. The sufficient condition obtained in the present paper for the collapse in the Bose system of particles $a_{\text {Born }}<0$ can be improved, and, thus, it is not necessary condition.

This work was supported by the National Academy of Sciences of Ukraine, Contract No. CC-10-2021.

1. V.L. Ginzburg, L.P. Pitaevskii. On the theory of superconductivity. Zh. Eksp. Teor. Fiz. 34, 1240 (1958) [in Russian].

2. E.P. Gross. Structure of a quantized vortex in boson systems. Nuovo Cimento 20, 454 (1961).

3. L.P. Pitaevskii. Vortex lines in the imperfect Bose-gas. $Z h$. Eksp. Teor. Fiz. 40, 646 (1961) [in Russian].

4. E.P. Gross. Hydrodynamics of a superfluid condensate. Math. Phys. 4, 195 (1963).

5. V.B. Bobrov, S.A. Trigger, A.G. Zagorodny. Nonstationary equation for one-particle wave function of the BoseEinstein condensate. Low Temperature Physics/Fizika Nizkikh Temperatur 47, 378 (2021).

6. L.P. Pitaevskii. Dynamics of collapse of a confined Bose gas. Phys. Lett. A 221, 14 (1996).

7. O.S. Davydov. Quantum Mechanics (Akademperiodyka, 2013) (in Ukrainian) [ISBN: 978-966-360-211-0].

8. A.G. Sitenko. Lectures in Scattering Theory (Pergamon, 1971) [ISBN: 978-0-08-016574-5].

9. F. Calogero. Variable Phase Approach to Potential Scattering (Academic Press, 1967) [ISBN: 978-966-360-211-0].

10. V.V. Babikov. The Method of Phase Functions in Quantum Mechanics (Nauka, 1976) (in Russian) [ISBN: 978966-360-211-0].

Received 04.11.21

Б.Є. Гринюк, К.О. Бугаєв

ЩОДО УМОВ ПРОСТОРОВОГО КОЛАПСУ В НЕСКІНЧЕННІЙ СИСТЕМІ БОЗЕ-ЧАСТИНОК

На основі варіаційного принципу показано, що умова просторового колапсу в бозе-газі не визначається величиною довжини розсіяння для потенціалу взаємодії між частинками, на відміну від результату, який випливає з рівняння Г роса-Пітаєвського і означає наявність колапсу у випадку від'ємної довжини розсіяння.

Kлючов $i$ слова: бозе-система, просторовий колапс. 